\title{
Collagen and elastin fibres
}

\author{
A. J. BAILEY \\ From the Agricultural Research Council, Meat Research Institute, Langford, Bristol
}

\begin{abstract}
Although an understanding of the intracellular biosynthesis of both collagen and elastin is of considerable importance it is the subsequent extracellular changes involving fibrogenesis and crosslinking that ensure that these proteins ultimately become the major supporting tissues of the body. This paper summarises the formation and stability of collagen and elastin fibres.
\end{abstract}

\section{Collagen}

The non-helical regions at the ends of the triple helix of procollagen probably provide a number of different intracellular functions-that is, initiating rapid formation of the triple helix; inhibiting intracellular fibrillogenesis; and facilitating transmembrane movement. They may also play an extracellular role (see D. S. Jackson, previous paper).

\section{PROCOLLAGEN-COLLAGEN CONVERSION}

The first extracellular step after secretion is the proteolytic cleavage of these $\mathrm{N}$ - and C-terminal nonhelical regions of procollagen (Bornstein, 1974). The actual location of cleavage of these extension peptides is not known, but they are probably removed during the formation of the fibre and may take part in fibrogenesis.

The intermediates in the conversion of procollagen to collagen have been analysed in detail (Byers et al., 1975; Fessler et al., 1975; Hoffman et al., 1976). Davidson et al. (1977) have shown that the $\mathrm{NH}_{2}$ terminus is cleaved first followed by stepwise scission of the disulphide bonded-COOH terminal extensions. Little is known about the enzymes in the process, although the retention of the $\mathrm{NH}_{2}$-terminus in dermatosparaxis suggests that there may be at least two different proteases. Until these enzymes have been isolated and tested against procollagen the precise mechanism is unlikely to be elucidated.

It is assumed that type III procollagen is converted to collagen in vivo by an analogous series of reactions, since native type III collagen molecules have been extracted from skin (Timpl et al., 1975). However, Goldberg (1977) found no evidence of conversion of type III procollagen to insoluble collagen whereas in the same fibroblast cultures native collagen was generated from type I procollagen. Whether this means that the two procollagens are converted by different enzyme systems and the type III enzyme was deficient in these fibroblast cultures, or that the processing of pro type III is extremely slow, is not known. The latter proposal is consistent with the higher proportion of soluble pro type III extractable from tissue (Lenaers and Lapiere, 1975; Timpl et al., 1975).

Basement membrane collagens, on the other hand, do not form fibres and this property may be due to the retention of the non-helical extension peptides (Kefalides, 1973). In-vivo biosynthetic studies showing the absence of any extension peptide removal support this (Minor et al., 1976), but other workers have reported that there is some cleavage of these peptides (Grant et al., 1975). It is generally agreed that in vivo procollagen remains in solution but that after removal of the extension peptides the properties of the molecule are drastically altered: it spontaneously precipitates to form fibrils. It is interesting to speculate that this multi-step processing of the procollagen has sone control function in which each discrete stage plays a part in the formation of a precisely organised fibre of uniform diameter.

FIBRILLAR ORGANISATION

The self-assembly of the processed molecules results in their highly ordered alignment into a fibril maintained by non-covalent bonds, both ionic and hydrophobic. The organisation of the processed collagen molecules within the microfibrils has been studied by a number of groups by electron microscopy and $x$-ray diffraction techniques (Traub and Piez, 1975; Ramachandran and Ramakrishnan, 1976; Miller, 1976).

Several models have been proposed. The pentafibril model, originally proposed by Smith (1968), has received considerable support from the $x$-ray diffraction studies of Miller and Wray (1971). This model incorporates the quarter-stagger and overlap hypothesis of Hodge and Petruska (1963), and at present is the best working model.

Once the lateral aggregation of the molecules into the fibrill has been attained the next questions are 
(1) How do the small fibres present in young tissue aggregate further to form the large diameter fibres observed in older tissue? and (2) What controls the ultimate fibre diameter, apparently fairly specific for different tissues-for example, about $20 \mathrm{~nm}$ in cornea and $200 \mathrm{~nm}$ in skin? The subsequent organisation of the fibres at the morphological level-for example, as laminates in cornea and at random in dermis-is presumably under cellular control, but no significant experiments to clarify this suggestion have been carried out.

\section{POLYMORPHIC FORMS OF COLLAGEN}

Genetically distinct types of collagen have been characterised and are now readily distinguishable (Miller, 1976). Apart from the amorphous basement membranes (type IV), the native fibrous structure of types I, II, and III, judged by electron microscopy, appear to be identical (Wiedemann et al., 1975). Subtle structural variations may be present which could result in functional differences for each fibre type. Support for this suggestion is slight at present; but the collagens are to some extent tissue specific and do not form mixed fibres. Bone and tendon are almost exclusively large type I fibres, cartilage solely type II fibres, and the fine reticulin fibres appear to be type III collagen. As the characterisation of the various polymorphic forms of collagen becomes more firmly established, it may be possible to relate the primary structural differences to their functional roles.

\section{CROSS-LINKING}

Although the precise alignment of the molecules in the fibrous collagen is maintained by ionic and hydrophobic bonding this fibre has no tensile strength. The mechanical stability of the collagen fibre is dependent on the subsequent formation of a series of covalent cross-links between the molecules making up the fibre. The chemistry of the cross-links has recently been reviewed (Bailey et al., 1974; Tanzer, 1976) and I will comment only on recent data and give an overall summary.

The initial reaction is the oxidative deamination of specific lysine and hydroxylysine residues located in the 15-20 amino-acid residues remaining of the non-helical regions at the $\mathrm{N}$ - and C-terminal ends of the molecules. Siegel (1974) showed that the lysyl oxidase does not act on tropocollagen molecules but only on the insoluble fibre aggregate formed under physiological conditions. A change from solution to the insoluble state is a biological requirement for cross-linking. Presumably the alignment of the molecules results in a local concentration of specific groups. The apposition of molecules in this conformation permits both binding of the lysyl oxidase and the subsequent spontaneous cross-linking of the resulting lysine-aldehydes. Since only the $\mathrm{N}$ - and $\mathrm{C}$ terminal lysine or hydroxylysine residues are affected the binding site of the enzyme is presumably on the molecule in the fibril adjacent to these groups-that is, the overlap position at $27 \mathrm{~nm}$ from either end of the molecule (residues 103 and 943). The amino-acid sequences of the $a$-chains around these sites are very similar in type I and type II collagens-that is, HypGly-His-Arg (Butler et al., 1976). Further, the only two histidine residues in the helical portion of the molecule occur at these two locations. Hence it is tempting to suggest that these residues take part in the enzymic process-for example, by stabilising the substrate-enzyme complex by proton donation as in the case of enzymes such as aldolase:

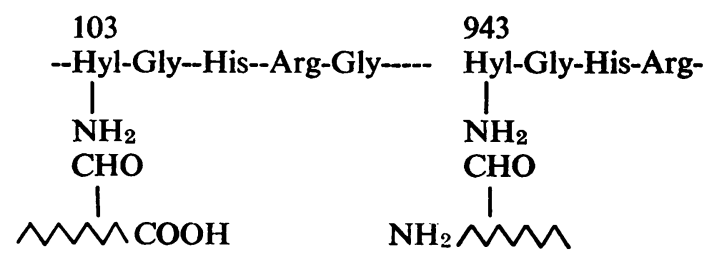

The subsequent condensation of the lysine and hydroxylysine aldehydes is generally believed to be spontaneous and non-enzymatic. The condensation products have been detected by reduction with tritiated borohydride, acid hydrolysis, and identification on an amino-acid analyser (Robins, 1976). From the structure obtained the nature of the invivo cross-link has been deduced to be the nonreduced form. Although a number of components have been isolated and characterised only three are present to any significant extent in the tissues studied to date. The two main reduced compounds isolated from young skin are hydroxylysinonorleucine (Bailey and Peach, 1968), derived from the condensation of allysine and hydroxylysine, and histidinohydroxymerodesmosine, derived from the condensation of the allysine aldol, hydroxylysine, and histidine (Tanzer et al., 1973). Although present in borohydride-reduced collagen, the presence in vivo of the non-reduced form of the latter has been disputed and is believed to be an artifact of the borohydride reduction (Robins and Bailey, 1973a). The main cross-link stabilising newly synthesised skin is therefore the aldimine bond, dehydrohydroxylysinonorleucine.

The predominant reducible component in bone and cartilage is hydroxylysinohydroxynorleucine, originally thought to be derived from the reduction of the aldimine dehydro-hydroxylysinohydroxynorleucine. But it has now been shown that the aldimine spontaneously undergoes an Amadori rearrangement and the reducible component acting- 
as the cross-link in vivo is hydroxylysino-5-ketonorleucine (Robins and Bailey, 1973b). The difference in the type of cross-link in skin and bone is therefore dependent on the extent of hydroxylation of the lysine residues in the $\mathrm{N}$ - and $\mathrm{C}$-terminal telo-peptides. The Amadori rearrangement results in a greater chemical stability of this bond, and this has permitted the isolation of a cross-linked peptide from non-borohydride-reduced cartilage collagen, confirming the existence of this cross-link in vivo.

Recently, two stable lysine-derived cross-links have been isolated from collagen, both directly from acid hydrolysates without borohydride reduction. Hydroxyaldol-histidine has been isolated from bovine skin but the reactions in its formation, including in-situ reduction, remain to be elucidated (Housley et al., 1975). A second presumptive crosslink has been isolated by Fujimoto et al. (1977). The formation of this cross-link also involves some unusual reactions, two allysine and one lysine residue condensing to form a pyridinium derivative. The relative importance of these putative cross-links and their relationship to age changes has not yet been shown. As with all the other cross-linking components their function as cross-links must be confirmed by isolating cross-linked peptides. The possibility of artefactual formation of supposed cross-linking compounds during isolation must also be eliminated.

\section{LOCATION OF CROSS-LINKS}

The isolation of cross-linked cyanogen bromide peptides from non-reduced cartilage located the cross-link between the $\mathrm{N}$-terminal telopeptide of one molecule and the overlap region at the C-terminal end of an adjacent molecule (Miller and Robertson, 1973). Type I peptides, owing to the presence of the a 2 chain, are more complex; but studies (Kang, 1972) have generally confirmed the end-overlap of the molecule predicted by the quarter-stagger theory. The basic cross-linking mechanism therefore appears to involve the aldehydes of the $\mathrm{N}$ - and $\mathrm{C}$ terminal non-helical regions in forming head-totail polymers. In older collagenous tissue, however, aldehydes do occur within the helical part of the the molecule (Deshmukh and Nimni, 1971), and, more recently, the isolation of cross-linked peptides believed to be derived from the helical regions has been reported (Fujii et al., 1975; Scott et al., 1976).The helical-helical cross-linked peptides have been isolated from bone and dentine and the inability of these tissues to swell has been attributed to this type of cross-link. The possibility of aldehyde in the helical region of the molecule raises considerable problems in the mechanism of cross-linking; hence the characterisation of these peptides must be confirmed.

\section{AGE-RELATED CHANGES}

That the physical properties of collagen change with age is generally agreed. Embryonic skin is less solubje than that of the young adult and analysis of the nature of the cross-links shows that embryonic skin contains the stable 'keto' cross-link, which accounts for its decreased solubility. There is a gradual change over to the labile aldimine crosslink towards the end of the gestation period and shortly after birth. This change in cross-links does not occur in all tissues. With tendon the proportion of the aldimine increases until it is equal to the 'keto' form whereas in bone and dentine little change occurs, the 'keto' form remaining the predominant cross-link (Bailey and Robins, 1973).

During maturation further changes occur. The fibres increase in tensile strength, become less soluble, and more resistant to chemical and enzymatic attack. These changes can be interpreted in terms of an alteration of the cross-linking of the molecules. Indeed the reducible cross-links, predominant in young tissue, decrease during maturation, leading to the suggestion that they are intermediates and that they are converted to a non-reducible form (Bailey et al., 1974).

The chemical nature of these non-reducible crosslinks has not yet been elucidated. It is now generally agreed that in-vivo reduction is not an operative mechanism. We have provided preliminary evidence that stabilisation occurs through an oxidative process (Bailey et al., 1977). This is clearly a function of normal maturation and should not be considered as a deleterious ageing process. The presence of reducible cross-links generally indicates the synthesis of new collagen. Hence the rate of disappearance of reducible cross-links with age will depend on the turnover rate of a particular tissue. For example, the in-vivo disappearance of the reducible cross-links is slow in bone because of its relatively high turnover rate but fast in lens capsule and cornea since the eye matures rapidly.

Although the two reducible cross-links can be considered labile or stable, this is a chemical concept. Both cross-links are stable under physiological conditions and the chemical differences in stability may be immaterial or solely a consequence of postribosomal levels of hydroxylation of the telopeptide lysine. However, the possibility that the stability of the cross-linking may be important in catabolism cannot be overlooked. Similarly, the physiological consequences of the conversion of the labilereducible to stable, non-reducible cross-links are not clear, although the changes in chemical and physical properties are readily demonstrable. It is therefore impossible at present to correlate cross-link stability with physiological function. 
The elucidation of the complete mechanism of stabilisation of the collagen fibre remains one of the major problems in collagen research. Furthermore, an explanation for the change in type of cross-link in embryonic dermis, with a second change during maturation, is clearly desirable. The artificial criteria by which we demonstrate differences in stability of the cross-link may not be of physiological importance.

\section{Elastin}

Elastic tissues of the body owe their mechanical properties to the protein elastin. In complete contrast to the highly orientated, inextensible collagen fibre the elastin fibre occurs naturally in a contracted state and is capable of reversible extension to about double its length. Elastin is therefore generally found in the form of fibres. It is also found as membranes in the elastic ligaments, elastic blood vessels, and other compliant tissues such as lung and skin. The elastic arteries contain concentric layers of elastic fibres, and the ligaments have parallel fibres (Partridge, 1962).

Both electron microscopy and $x$-ray diffraction have shown elastin to be basically an amorphous matrix. More recently high resolution electron microscopy together with optical diffraction of the micrographs has provided evidence for some fibrillar order by showing the presence of parallel filaments 3-4 $\mathrm{nm}$ in diameter with a periodicity of $4 \mathrm{~nm}$ (Gotte et al., 1974).

Elastin was at first defined solely by its histological appearance. Largely through the work of Partridge and his colleagues, a precise chemical definition of elastin was reported in 1958. However, it was not until the cross-links were identified by this group in 1963 (Thomas et al., 1963) that the field opened up and a significant understanding of the relationship of structure to function began to emerge.

Like collagen, elastin is an extracellular insoluble polymeric protein; hence its intracellular biosynthesis as a soluble monomer, its extracellular aggregation and subsequent stabilisation by crosslinking considerably resemble the biosynthesis of collagen fibres.

\section{BIOSYNTHESIS}

As in the case of collagen it has always been thought that there must be a soluble monomer synthesised within the cell but incapable of aggregation. The discovery of procollagen possessing just such properties renewed efforts to identify proelastin. Unfortunately, studies of the early stages of elastin biosynthesis have been hampered by the absence of a reliable marker for this protein.
The first real insight into the biosynthesis of elastin came when a soluble protein closely resembling elastin was isolated from the aortas of copperdeficient pigs (Smith et al., 1972). An important aspect of the purification was the use of a coacervation step. This ability of soluble elastin to come out of solution was first demonstrated by Partridge and his coworkers (Partridge et al., 1955) using oxalic acid-solubilised intact elastin. The amino-acid composition of the putative tropoelastin was similar to insoluble elastin except for a high lysine content and a low level of the cross-linking amino-acids desmosine and isodemosine, known to be derived from lysine. The molecular weight was shown to be about 74000 -that is, about 880 residues, $85 \%$ of which were non-polar.

Although apparently amorphous in structure compared to fibrous collagen the coacervate seems to have some structure. Circular dichroism revealed a considerable amount of $a$-helical configuration (Urry et al., 1969), and the presence of a fibrillar structure was revealed by negative staining in the electron microscope (Cox et al., 1973). The extent of this structural organisation is still the subject of intensive studies. The important point in biosynthesis is the ability of the tropoelastin to come out of solution under physiological conditions, analogous to the precipitation of collagen. This property strengthened the case for the formation of a precursor containing extension peptides-that is, proelastin-soluble under physiological conditions as the initial step in the cellular synthesis.

\section{PROELASTIN}

Franzblau and his colleagues have isolated a high molecular weight soluble elastin which they believe to be the precursor, proelastin (Foster et al., 1977). By ensuring the inactivation of proteolytic enzymes, a neutral salt extract of lathyritic chick aorta was found to contain a homogeneous protein of 130000 daltons. The protein cross-reacted with sera against chick tropoelastin but on immunoelectophoresis gave a precipitin line separate from tropoelastin. The amino-acid sequence of the $\mathrm{N}$-terminal region was found to be similar to tropoelastin, suggesting that the extension peptide must be at the $\mathrm{C}$-terminal end. This group of workers suggested that fibrogenesis occurs through association of the extension peptides via disulphide bridges to other molecules in the microfibrils. This would present an insoluble substrate to the lysyl oxidase and subsequent cross-link formation.

This hypothesis does not take cognisance of the ability of soluble elastin to coacervate. An alternative mechanism analogous to the procollagen-collagen conversion would be cleavage of the extension pep- 
tides and spontaneous coacervation of the tropoelastin. In contrast to the fibrous precipitate obtained with collagen the elastin coacervate is basically amorphous. However, sufficient structure must exist for interaction with lysine oxidase and the subsequent formation of cross-links at precise locations in the polymer. Evidence for a partially organised structure for tropoelastin has been discussed above.

Other workers have failed to find proelastin. Narayanan (1976) found two components of 150000 and 74000 daltons but showed that the higher molecular weight component was collagen not elastin. Similarly, using matrix-free cells in the presence of protease inhibitors, Bressan and Prockop (1977) found only elastin as a 72000 -dalton component. Using a different approach Sykes and Hawker (1977) used anti-soluble elastin sera to follow the molecular weight distribution of elastin products from cell cultures. They failed to find the theoretical precursor.

The existence of a precursor form has been deduced from the studies on collagen and from the fact that tropoelastin coacervates under physiological conditions. Its existence remains to be confirmed by other workers, and the evidence for its identity must be conclusive.

\section{TROPOELASTIN}

Having established the similarity of tropoelastin to native elastin it clearly had to be shown that it was not a degradation product but exhibited a precursorproduct relationship through its conversion to insoluble elastin by formation of the desmosine cross-links. The in-vitro incorporation of ${ }^{3} \mathrm{H}-4 \cdot 5$ lysine with cultured aorta from copper deficient swine demonstrated that the elastin rapidly became insoluble and the ${ }^{3} \mathrm{H}$-labelled cross-links desmosine and isodesmosine were identified (Smith et al., 1975). Similar studies have also been carried out with cultured cells.

\section{PRIMARY STRUCTURE}

Not until tropoelastin had been isolated was it possible to obtain meaningful data on the primary structure of elastin. Sandberg et al. (1971) obtained sequences of tropoelastin peptides from copperdeficient swine derived from material digested with trypsin, subtilisin, and thrombin. Most extensively investigated have been the tryptic peptides. They fall into two distinct groups-those of over 20 residues (14 peptides equivalent to 684 residues) and those of four fewer residues ( 21 peptides equivalent to 66 residues). The molecule seems to contain $850-870$ residues, giving a molecular weight of 72-74 000 daltons. The striking feature of the large peptides is that they contain repeating sequences of the peptides Pro-Gly-Gly-Val; Pro-Gly-Val-GlyVal; and Gly-Val-Gly-Val-Ala. Such repeating sequences imply order that should be reflected in the tertiary structure.

Synthetic peptides with this type of sequence have been studied by Urry and his coworkers (Urry and Long, 1976). Each has a preferred secondary structure, giving further support to the idea that elastin is not a random chain. The peptides were found to contain a conformational feature called the beta-turn which uses Pro-Gly residues to produce almost right-angle bends in the peptide chain. Much of this work has been carried out in organic solvents, but Urry and Long (1976) suggest that under aqueous physiological conditions the conformation is more dynamic. This polypentapeptide shows a very similar ${ }^{13} \mathrm{C}$ magnetic resonance spectrum to $a$-elastin, suggesting that it may well be a good model for elastin.

\section{CROSS-LINKING OF ELASTIN}

The principles of the cross-linking of elastin were established by Partridge and his coworkers (Partridge, 1966a). Subsequent studies have shown that the cross-linking of collagen is by the same basic mechanism, but knowledge of the reactions in elastin are more complete than for collagen.

The initial stage is the oxidative deamination of the $\epsilon-\mathrm{NH}_{2}$ group of specific lysine residues by lysyl oxidase, a copper metallo-protein oxidase. The enzyme can be obtained by urea extractions from chick cartilage and purified by affinity chromatography using a column of collagen bound to sepharose. The molecular weight of the enzyme is $\mathbf{4 0} 000$ daltons and it seems to be equally effective on both collagen and elastin (Siegel, 1974). Lysyl oxidase is specifically inhibited by deoxygenation and by the nitrils that produce lathyrism - that is, the feeding of these compounds to animals inhibits the biosynthesis of the cross-links, resulting in an extreme fragility of the connective tissues. Since the enzyme contains copper, chronic copper deficiency results in a condition similar to experimental lathyrism.

During the oxidative deamination of tropoelastin there is a large-scale conversion of the lysine residues from about 40 down to 7 residues per thousand. Tropoelastin contains few polar residues, and this decrease in charge must have a profound effect on the structure of the molecules in the fibre during the cross-linking process. The point of attack of the enzymes has not yet been determined but from the gross configurational changes occurring on coacervation one might expect the enzyme to attack at this stage, the requisite lysines from adjacent molecules then being in the correct apposition. Preliminary data supporting this suggestion have been reported 
by Narayanan et al. (1976), who showed that the lysyl oxidase was about 10 times less effective in producing the desmosines from tropoelastin in solution at $11^{\circ} \mathrm{C}$ than as a coacervate at $37^{\circ} \mathrm{C}$. This is analogous to the inability of lysyl oxidase to react with tropocollagen compared to the insoluble fibre and again suggests that the coacervate has sufficient structure to provide specific binding sites to locate the enzymes with the oxidizable lysines.

The cross-links in mature elastin fibres have been well characterised but only recently has the biosynthesis of the desmosine and isodesmosine from tropoelastin been demonstrated.

After allysine is formed the putative intermediates, the aldol condensation product, dehydrolysinonorleucine and dehydromerodesmosine, are found. In the formation of the stable cross-links desmosine and isodesmosine condensation of the above intermediates occurs to form the 1:2 dihydropyridines. The latter are subsequently oxidised to the desmosines. This oxidation step may take place spontaneously, as proposed by Davis and Anwar (1970), or by reacting with dehydrolysinonorleucine, as suggested by Piez (1968). Indeed, lysinonorleucine has been identified in elastin (Lent and Franzblau, 1967) although in insufficient quantities to account for the number of desmosines. The lysinonorleucine content is about one per thousand residues compared with 3-5 desmosines per thousand, suggesting that an additional mechanism must be operative. Alternatively, an additional enzyme could be involved, although in-vitro incubation studies of pure enzyme and tropoelastin, when the desmosines are formed without additional cofactors, make this unlikely. Furthermore, desmosines are formed at the same rate in the absence of molecular oxygen. On the available evidence it seems that the cross-linking reactions are spontaneous chemical reactions of groups placed in a specific, highly concentrated environment and, as in the case of collagen, probably require no further factors.

Although most of the products have been identified, the precise pathway of desmosine synthesis remains to be elucidated. The allysine residue could react with dehydromerodesmosine, or the aldol condensation product in one chain could react with dehydrolysinonorleucine in another. The problem will probably have to be answered from the sequence of cross-linked peptides. It is noteworthy that elastin from lathyritic chick aorta (Sykes and Partridge, 1974) and elastin synthesised by smooth muscle cells in vitro (Faris et al., 1976) are insoluble in hot alkali yet contain few desmosine residues. This suggests that the insolubility may be due to the aldol, indicating that it must be an inter- rather than an intramolecular cross-link. On the other had, a very small number of desmosines could confer insolubility.

\section{LOCATION OF CROSS-LINKS}

Almost certainly there are precisely ordered regions for the cross-linking areas. This seems necessary for lysyl oxidase specificity, its facility of access, and the need for the subsequent condensation reactions to form desmosine and isodesmosine in a controlled manner. Despite elastin's random structure the cross-link must be precisely located.

Support for these suggestions is provided by the recent primary sequence data in which the lysine residues are seen to be in a highly unusual arrangement. They seem to occur in pairs as alanine-rich $a$-helical regions, in two main types of sequence, in porcine tropoelastin (Sandberg et al., 1971):

\section{I -Ala-Ala Lys Ala Ala Lys Tyr Gly Ala- \\ II -Ala Ala Lys Ala Ala Ala Lys Ala Ala-}

Based on stereochemical arguments, Gray et al. (1973) proposed that the type I sequence gave rise to dehydrolysinonorleucine and the type II to the aldol condensation product. Condensation of two intermediates would give rise to the dihydrodesmosines which produce desmosines on oxidation. They further suggested that the Tyr residue in type I serves to protect the preceding lysine from oxidation by lysyl oxidase and as a possible electron carrier to oxidise the dihydrodesmosines. A similar analysis of bovine elastin by Gerber and Anwar (1975) revealed phenylalanine at this location. This residue could prevent enzymic oxidation but would not act as an oxidising agent. Clearly, primary sequence studies of tropoelastin are providing valuable data on possible cross-link locations, but definite conclusions must await analysis of the crosslinked peptides themselves.

Preliminary data on the sequence of desmosinecontaining peptides have been obtained. Since crosslinked peptides must of necessity involve sequencing of the two chains simultaneously, both chains containing polyalanine sequences, the assignments are tenuous. Despite the difficulties Franzblau and his colleagues (Foster et al., 1974) have interpreted the sequence of two peptides with the basic core around the desmosine:

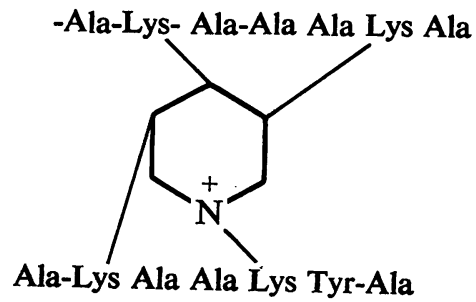


Although the desmosines are tetrafunctional, the sequence data seem to indicate that they cross-link only two chains.

STRUCTURE-FUNCTION RELATIONSHIP The physicochemical studies of elastin have clearly shown that elastin, in contrast to the inextensible crystalline structure of collagen, is amorphous and highly elastic. Elastin exhibits properties typical of amorphous polymers; it is a soft, rubbery solid when wet but a brittle glassy solid when dry. It has an amorphous $x$-ray diffraction pattern.

The energy to restore the shape of the deformed material in elastic materials can come from two sources, enthalpy and entropy. The enthalpy term is overwhelmingly predominant in materials like steel. The entropy term is of prime importance with rubbers.

Two views of the structure-function relationship of elastin have evolved. Put simply, the first, favoured by physicists, is that the polypeptide chains are randomly coiled and theoretically free, as in classical rubbers. The second, favoured by protein chemists, is that the chains have a preferred conformation that is always returned to after stretching.

From stress-temperature measurements, Hoeve and Flory $(1958,1974)$ account for the elasticity of elastin in terms of configurational entropy changes when an amorphous three-dimensional net is stretched. According to this classical rubber theory there is no interaction between chains and the crosslinks are randomly distributed. They conclude that elastin is devoid of any regular domains and that its molecular structure may be considered as a single phase liquid-like structure. These studies have been supported by others, particularly McCrum and coworkers (Dorrington et al., 1975).

An alternative to the random model was suggested by Partridge $(1966 \mathrm{~b}, 1977)$. He proposed that elastin is a two-phase system with hydrophobic groups stabilising the interior of globules of molecular weight of about 70000 . The few polar groups are arranged on the outside of these globules towards the water phase. This type of structure would look filamentous in the electron microscope owing to penetration of the stain between the line of globules. The model suggests a return to a preferred configuration after extension. It also suggests that overextension would be limited by cross-links at specific locations determined by the primary sequence to keep the chains in register. Elastin would not be a true elastomer on this basis but would also possess a small enthalpy term due to the organised part of the structure.

Weis-Fogh and Andersen (1970) subsequently attempted to explain the elasticity of such a model by assuming deformation of the globules into ellipsoids during stretching. To demonstrate whether elastin behaves according to the theory of rubbers or whether hydrophobic interactions are involved they measured with a sensitive calorimeter the heat produced during stretching and reabsorbed during relaxation. The heat produced during stretching always exceeded that corresponding to the chemical work done, indicating that the chemical changes in the deformation of elastin in water are reversible and must therefore differ fundamentally from rubbers. They proposed that this exposure of the hydrophobic groups to an aqueous environment would result in a considerable decrease in entropy of the system, and that the restoring force would therefore be larger than if solely due to configurational entropy, as in rubbers. A further modification of this model, the oiled-coil model, was proposed by Gray et al. (1973); this was made up of alternating segments of $\alpha$-helical cross-link regions and of an open left-handed coil that conferred longrange extensibility. The diameter of this fundamental filament would be $1-1.5 \mathrm{~nm}$-that is, about the same size as the filamentous units observed in the electron microscope.

Both Dorrington et al. (1975) and Hoeve and Flory (1974) pointed out that Weis-Fogh and Andersen had ignored a major source of heat release arising from the stress-induced increase in volume of the specimen. When this factor is taken into account the results agree with the entropy elastic deformation theory and do not support the Partridge globular model. On the other hand, Gosline (1975) measured the energy changes during stretching by microcalorimetric techniques and showed that the only significant process contributing to the internal energy is the absorption of water on hydrophobic groups. He concluded that all the elastic energy is stored in the reversible exposure of hydrophobic groups during stretching, as proposed by Weis-Fogh and Andersen (1974). However, he also concluded that these hydrophobic interactions take place within a totally random and kinetically free polymer network and do not indicate a degree of crystallinity.

We must remember that these models for elastin are based on particular types of physical measurement. Despite the fact that the random network model provides a best fit for the stress-strain measurements, it does not mean that this is the complete answer. Elastin has many unusual features when compared to rubber-for example, a soluble precursor with a specific repeating primary sequence; the ability to coacervate and form a fibrous structure, indicating strong hydrophobic interactions; and the capability of acting as a substrate for lysyl oxidase, 
indicating some structure in the substrate. It is difficult for protein chemists to accept the random chain model until these properties can be reconciled with this theory. The application of some alternative physicochemical techniques may prove valuable.

Some of the difficulties of these extreme views may arise from the solvent mixtures used by the physical chemists. For example, to avoid complicated mathematical corrections due to temperature changes arising from volume changes Hoeve and Flory (1974) used $30 \%$ ethylene glycol as a swelling agent, despite the fact that elastin seems to be sensitive to configurational changes brought about by temperature, solvent, $\mathrm{pH}$, and ionic strength. The importance of these factors has been shown by their dramatic effect on the coacervation of tropoelastin and $a$-elastin (Partridge and Whiting, 1977). Elastin in vivo is in a poor solvent and under these conditions chain interaction can occur in specific parts of the structure.

Some evidence of this nature has been obtained from ${ }^{13} \mathrm{C}$ magnetic resonance studies. The chain motion is apparently restricted in water compared with fibres swollen in polar organic solvents which suppress hydrophobic side-chain bonding. A detailed study by Lyerla and Torchia (1975) indicated that $80 \%$ of the elastin backbone carbons exhibited mobility similar to an amorphous polymer such as rubber. Furthermore, the motion of the alanine residues which are concentrated in the region of the cross-links was particularly restricted. The glycine residues occur in repeat sequences with proline and valine and it would be interesting to know if this technique could show whether the alanine-rich and glycine-rich regions had different molecular structures.

Urry and his group have studied polymers typical of the repeating sequence reported to be present in tropoelastin (Urry and Long, 1976). These polypeptides exhibited $\beta$-turns with proline and glycine in the second and third positions at the corners as dominant conformational features. The $\beta$-turn utilises a hydrogen bond between the $\mathrm{C}-\mathrm{O}$ of residue 1 and the N-H of residue 4. Despite the fact that these conformations were determined in dimethylsulphoxide and trifluoroethanol, Urry claims that under physiological conditions the structures would be more dynamic. Making the solvent more polar stabilises the secondary structure, possibly owing to the decreased activity of the water. In the same way saline, glycol, and the blocking of the charged lysine groups decreases but sharpens the temperature of coacervation.

The synthetic cross-linked polypeptides also exhibit rubber-like properties and can form filamentous structures. Yet they prefer conformations in which the $\beta$-turn is the key repeating unit, while the $\beta$-spiral conformation leads to the filamentous structures. Urry and his group do not claim that the structure is of ordered $a$-helices but that it is a dynamic structure having preferred interchain hydrophobic interactions.

Clearly this description of elastin possessing a filamentous ultrastructure and extensive hydrophobic interchain associations is inconsistent with the classical rubber elasticity theory of kinetically free chains. Urry makes the interesting calculation, based on the mobility of the chains from ${ }^{13} \mathrm{C}$ magnetic resonance, that if cross-linking results from random interchain contact in a completely random structure it would take $10^{40}$ years to achieve 20 cross-links per 70000 dalton units.

In summary, even though the structure of elastin may be fitted to a simple statistical theory the other studies certainly indicate that it is more complex and that it cannot be a true elastomer. There is no a priori reason to fit the structure of elastin into either extreme theory. Elastin may be unique.

\section{Conclusions}

Both collagen and elastin research have benefited from the realisation that defects in the formation of these fibres can be the basis of many pathological disorders. Indeed, molecular aberrations have already been reported for a number of heritable collagen diseases (see C. I. Levene (Levene, 1978) at page 82, and F. M. Pope and A. C. Nicholls (Pope and Nicholls, 1978) at page 95), although they still have to be correlated directly with functional impairment.

The identification of procollagen and the various polymorphic forms of the collagen molecules makes it possible confidently to predict similar phenomena in elastin. Despite the experimental difficulties, preliminary data indicate the presence of a proelastin. It is not unlikely that ligament, aorta, and lung possess polymorphic forms of elastin. These studies may also reveal molecular defects in elastin biosynthesis resulting in pathological disorders.

\section{References}

Bailey, A. J., and Peach, C. M. (1968). Isolation and structural identification of a labile inter-molecular cross-link in collagen. Biochemical and Biophysical Research Communications, 33, 812-819.

Bailey, A. J., and Robins, S. P. (1973). Development and maturation of the cross-links in the collagen fibres of skin. Frontiers of Matrix Biology, 1, 130-156.

Bailey, A. J., Robins, S. P., and Balian, E. (1974). Biological significance of the intermolecular cross-links of collagen. Nature (London), 251, 105-109.

Bailey, A. J., Ranta Helena M., Nicholls, A. C., Partridge, S. M., and Elsden, D. F. (1977). Isolation of $\alpha$-amino 
adipic acid from mature dermal collagen and elastin. Evidence for an oxidative pathway in the maturation of collagen and elastin. Biochemical and Biophysical Research Communications, 78, 1403-1409.

Bornstein, P. (1974). Biosynthesis of collagen. Annual Reviews of Biochemistry, 43, 567-603

Bressan, G. M., and Prockop, D. J. (1977). Synthesis of elastin in aortas from chick embryos. Biochemistry, 16, 1406-1412.

Butler, W. T., Miller, E. J., and Finch, J. E. (1976). The covalent structure of cartilage collagen. Biochemistry, 15, 3000-3006.

Byers, P. H., Click, E. M., Harper, E., and Bornstein, P. (1975). Interchain disulfide bonds in procollagen are located in a large non-triple-helical $\mathrm{COOH}$-terminal domain. Proceedings of the National Academy of Sciences of the United States of America, 72, 30093013.

Cox, B. A., Starcher, B. C., and Urry, D. W. (1973). Coacervation of $\alpha$-elastin results in fibre formation. Biochimica et Biophysica Acta, 317, 209-213.

Davidson, J. M., McEneany, L. S., and Bornstein, P. (1977). Intermediates in the conversion of procollagen to collagen. European Journal of Biochemistry, 81, 349-355.

Davis, N. R., and Anwar, R. A. (1970). On the mechanism of formation of desmosine and isodesmosine cross-links of elastin. Journal of the American Chemical Society, 92, 3778-3782.

Deshmukh, K., and Nimni, M. E. (1971). Characterisation of the aldehydes present on the cyanogen bromide peptides from mature rat skin collagen. Biochemistry, 10, 1640-1647.

Dorrington, K., Grut, W., and McCrum, N. S. (1975). Mechanical state of elastin. Nature (London), 255, 476-478.

Faris, B., Salcedo, L. L., Cook, V., Johnson, L., Foster, J. A., Franzblau, C. (1976). The synthesis of connective tissue protein in smooth muscle cells. Biochimica et Biophysica Acta, 418, 93-103.

Fessler, L. I., Morris, N. P., and Fessler, J. H. (1975). Procollagen: biological scission/of amino and carboxyl extension peptides. Proceedings of the National Academy of Sciences of the United States of America, 72, 4905-4909.

Foster, J. A., Mecham, R., Imberman, M., Faris, B., and Franzblau, C. (1977). A higher molecular weight species of soluble elastin-proelastin. Advances in Experimental Medicine and Biology, 79, 351-369.

Foster, J. A., Rubin, L., Kagan, H. M., Franzblau, C., Bruenger, E., and Sandberg, L. B. (1974). Isolation and characterisation of cross-linked peptides from elastin. Journal of Biological Chemistry, 249, 6191-6196.

Fujii, K., Corcoran, D., and Tanzer, M. L. (1975). Isolation and structure of a crosslinked tripeptide from calf bone collagen. Biochemistry, 14, 4409-4413.

Fujimoto, D., Akiba, K., and Nakamura, N. (1977). Isolation and characterisation of a fluorescent material in bovine achilles tendon. Biochemical and Biophysical Research Communications, 76, 1124-1129.

Gerber, G. E., and Anwar, R. A. (1975). Comparative studies of the cross-linked regions of elastin from bovine ligamentum nuchae and bovine, porcine, and human aorta. Biochemical Journal, 149, 685-695.

Goldberg, B. (1977). Kinetics of processing of Type I and Type III procollagens in fibroblast cultures. Proceedings of the National Academy of Sciences of the United States of America, 74, 3322-3325.

Gosline, J. M. (1975). The physical properties of elastic tissue. International Review of Connective Tissue Research, 7, 211-249.

Gotte, L., Giro, M. G., Volpin, D., and Horne, R. W. (1974). The ultrastructural organisation of elastin. Journal of Ultrastructure Research, 46, 23-33.

Grant, M. E., Harwood, R., and Williams, I. (1974). The biosynthesis of glomerular basement membrane collagen. Biochemical Society Transactions, 2, 624-626.

Gray, W. R., Sandberg, L. B., and Foster, J. A. (1973). Molecular model for elastin structure and function. Nature (London), 246, 461-466.

Hodge, A. J., Petruska, J. H. (1963). In Aspects of Protein Structures, p. 289. Academic Press, New York.

Hoeve, C. A. J., and Flory, P. J. (1958). The elastic properties of elastin. Journal of the American Chemical Society, 80, 6523-6526.

Hoeve, C. A. J., and Flory, P. J. (1974). The elastic properties of elastin. Biopolymers, 13, 677-686.

Hoffman, H. P., Olsen, B. R., Chen, H. T., and Prockop, D. J. (1976). Segment-long-spacing aggregates and isolation of $\mathrm{COOH}$-terminal peptides from Type I procollagen. Proceedings of the National Academy of Sciences of the United States of America, 73, 4304-4308.

Housley, T. J., Tanzer, M. L., Henson, E., and Gallop, P. M. (1975). Collagen cross-linking: isolation of hydroxyaldol-histidine, a naturally-occurring crosslink. Biochemical and Biophysical Research Communications, 67, 824.

Kang, A. H. (1972). Studies on the location of intermolecular cross-links in collagen. Isolation of a $\mathrm{CNBr}$ peptide containing $\delta$-hydroxylysinonorleucine leucine. Biochemistry, 11, 1828-1835.

Kefalides, N. A. (1973). Biosynthesis and structure of basement membranes. International Review of Connective Tissue Research, 6, 63-104.

Lenaers, A., and Lapiere, C. M. (1975). Type III procollagen and collagen in skin. Biochimica et Biophysica Acta, 400, 121-131.

Lent, R., and Franzblau, C. (1967). Studies on the reduction of bovine elastin: evidence for the presence of $\triangle^{6: 7}$-dehydrolysinonorleucine. Biochemical and Biophysical Research Communications, 26, 43-50.

Levene, C. I. (1978). Diseases of the collagen molecule Journal of Clinical Pathology, 31, Supplement (Royal College of Pathologists) 12, 82-94.

Lyerla, J. R., and Torchia, D. A. (1975). Molecular mobility and structure of elastin deduced from the solvent and temperature dependence of ${ }^{13} \mathrm{C}$ magnetic resonance relaxation data. Biochemistry, 14, 5175-5183.

Miller, A. (1976). Molecular packing in collagen fibrils. In Biochemistry of Collagen, edited by G. N. Ramachandran and A. H. Reddi, pp. 85-117. Plenum, New York.

Miller, A., and Wray, J. S. (1971). Molecular packing in collagen. Nature (London), 230, 437-439. 
Miller, E. J. (1976). Biochemical characteristics and biological significances of the genetically distinct collagens. Molecular and Cellular Biochemistry, 13, 165-192.

Miller, E. J., and Robertson, P. B. (1973). The stability of collagen crosslinks when derived from hydroxylysyl residues. Biochemical and Biophysical Research Communications, 54, 432-439.

Minor, R. R., Clark, C. C., Strause, E. L., Koszalka, T. R., Brent, R. L., and Kefalides, N. A. (1976). Basement membrane procollagen is not converted to collagen in organ cultures of parietal yolk sac endoderm. Journal of Biological Chemistry, 251, 1789-1794.

Narayanan, A. S., Page, R. C., and Kuzan, F. (1977). Studies on the action of lysyl oxidase on soluble elastin. Advances in Experimental Medicine and Biology, 79, 491-508.

Partridge, S. M., Davis, H. F., and Adair, G. S. (1955). The Chemistry of connective tissues 2 . Soluble proteins derived from partial hydrolysis of elastin. Biochemical Journal, 61, 11-21.

Partridge, S. M. (1962). Elastin. Advances in Protein Chemistry, 17, 227-297.

Partridge, S. M. (1966a). Biosynthesis and nature of elastin structure. Federation Proceedings, 25, 1023-1029.

Partridge, S. M. (1966b). Elastin. In The Physiology and Biochemistry of Muscle as Food, edited by E. J. Briskey, R. G. Cassens, and J. C. Trautman, pp. 327339. University of Wisconsin Press, Madison.

Partridge, S. M. (1977). The Lability of elastin structure and its probable form under physiological conditions. Frontiers of Matrix Biology, in press.

Partridge, S. M., and Whiting, A. M. (1977). The coacervate-sol transition observed with $\alpha$-elastin and its $\mathrm{N}$-formyl O-methyl derivative. Advances in Experimental Medicine and Biology, 79, 715-723.

Piez, K. A. (1968). Crosslinking of collagen and elastin. Annual Review of Biochemistry, 37, 547-567.

Pope, F. M., and Nicholls, A. C. (1978). Molecular abnormalities of collagen. Journal of Clinical Pathology. 31, Supplement (Royal College of Pathologists) 12, 95-104

Ramachandran, G. N., and Ramakrishnan, C. (1976). Molecular structure. In Biochemistry of Collagen, edited by G. N. Ramachandran and A. M. Reddi, pp. 45-81. Plenum, New York.

Robins, S. P. (1976). The separation of cross-linking components from collagen. In Methodology of Connective Tissue Research, edited by D. A. Hall, pp. 37-52. Joynson-Bruvvers, Oxford.

Robins, S. P., and Bailey, A. J. (1973a). The chemistry of the collagen cross-links: fraction $\mathrm{C}$, possible artifact produced during the reduction of collegan fibres with borohydride. Biochemical Journal, 135, 657-665.

Robins, S. P., and Bailey, A. J. (1973b). Relative stabilities of the intermediate reducible crosslinks present in collagen fibres. FEBS Letters, 33, 167-171.

Sandberg, L. B., Weismann, N., and Gray, W. (1971). Structural features of tropoelastin related to the sites of cross-links in aortic elastin. Biochemistry, 10, 52-56.

Scott, P. G., Veis, A., and Mechanic G. (1976). The identity of a cyanogen bromide fragment of bovine dentine collagen containing the site of an intermolecular cross-link. Biochemistry, 15, 3191-3198.

Siegel, R. C. (1974). Biosynthesis of collagen cross-links. Increased activity of purified lysyl oxidase with reconstituted collagen fibrils. Proceedings of the National Academy of Sciences of the United States of America, 71, 4826-4830.

Smith, J. W. (1968). Molecular pattern in native collagen. Nature (London), 219, 157-158.

Smith, D. W., Brown, D. M., and Carnes, W. H. (1972). Preparation and properties of salt-soluble elastin. Journal of Biological Chemistry, 247, 2427-2432.

Smith, D. W., Abraham, P. A., and Carnes, W. M. (1975). Cross-linkage of salt-soluble elastin in vitro. Biochemical Biophysical Research Communications, 66, 893-899.

Sykes, B. C., and Partridge, S. M. (1974). Salt-soluble elastin from lathyritic chicks. Biochemical Journal, 141, 567-572.

Sykes, B. C., and Hawker, S. (1977). Some investigations of elastin biosynthesis in vitro using an immunoprecipitate. Advances in Experimental Medicine and Biology, 79, 453-459.

Tanzer, M. L. (1976). Cross-linking in Biochemistry of Collagen, edited by G. N. Ramachandran and A. H. Reddi, pp. 137-157. Plenum, New York.

Tanzer, M. L., Housley, T., Berube, L., Fairweather, R., Franzblau, C., and Gallop, P. (1973). Structure of two histidine-containing cross-links from collagen. Journal of Biological Chemistry, 248, 393-402.

Thomas, J., Elsden, D. F., and Partridge, S. M. (1963) Partial structure of the major degradation products from the cross-linkage in elastin. Nature (London), 200,651-652.

Timpl, R., Glanville, R. W., Nowack, H., Weidmann, H., Fietzek, P., and Kuhn, K. (1975). Isolation, chemical and electron microscopical characterization of neutral salt soluble Type III collagen and procollagen from fetal bovine skin. Hoppe-Seylers Zeitschrift für Physiologische Chemie, 356, 1783-1792.

Traub, W., and Piez, K. A. (1971). The Chemistry and structure of collagen. Advances in Protein Chemistry, $25,243$.

Urry, D. W., Starcher, B. C., and Partridge, S. M. (1969). Coacervation of solubilized elastin effects a notable conformational change. Nature (London), 222, 795-796.

Urry, D. W., and Long, M. N. (1976). Conformations of the repeat peptides of elastin in solution. An application of proton and carbon-13 magnetic resonance to the determination of polypeptide secondary structure. Critical Reviews in Biochemistry, 4, 1-45.

Weis-Fogh, T., and Andersen, S. O. (1970). New molecular model for the long-range elasticity of elastin. Nature (London), 227, 718-721.

Wiedemann, H., Chung, E., Fujii, T., Miller, E. J., and Kuhn, K. (1975). Comparative electron microscope studies on Type III and Type I collagens. European Journal of Biochemistry, 51, 363-368. 